\title{
Pathologies sociales de la communication
}

\section{Pascal Durand}

\section{OpenEdition}

\section{Journals}

Édition électronique

URL : http://journals.openedition.org/questionsdecommunication/430

DOI : 10.4000/questionsdecommunication.430

ISSN : 2259-8901

\section{Éditeur}

Presses universitaires de Lorraine

\section{Édition imprimée}

Date de publication : 1 juillet 2009

Pagination : 7-19

ISBN : 978-2-86480-989-0

ISSN : 1633-5961

\section{Référence électronique}

Pascal Durand, «Pathologies sociales de la communication », Questions de communication [En ligne], 15 | 2009, mis en ligne le 04 janvier 2012, consulté le 19 avril 2019. URL : http:// journals.openedition.org/questionsdecommunication/430; DOI : 10.4000/ questionsdecommunication.430 


\section{$>$ DOSSIER}

PASCAL DURAND

Institutions culturelles et information

Université de Liège

pascal.durand@ulg.ac.be

\section{PATHOLOGIES SOCIALES DE LA COMMUNICATION}

$\mathrm{D}$ e la communication, souligner non les vertus, mais les vices ; examiner, au titre de ses pathologies - et plus spécialement de ses pathologies sociales -, non les biais dont elle peut faire l'objet, mais ceux à la source desquels il arrive qu'elle se trouve ; l'envisager, non comme facteur de prévention ou de résorption de ces pathologies, mais plutôt comme leur éventuel vecteur d'aggravation circulaire, voilà comment pourrait être résumé à grands traits le propos du dossier ouvert dans la présente livraison de Questions de communication. Programme exorbitant : quelques articles réunis pour la cause ne sauraient épuiser ni même baliser avec précision une problématique pouvant aller, de façon très reçue, des phénomènes d'addiction électronique et des syndromes de déréalisation ou d'isolement induits par les technologies du virtuel aux dispositifs de propagande et autres techniques de lissage des différends politiques ou sociaux. Programme qui n'en paraîtra pas moins incongru au regard des conceptions enchantées de la communication et de la doxa qui l'institue non seulement en principe actif de la vie sociale en général, mais encore en ressource de première nécessité et en valeur indiscutable.

La notion de « pathologie sociale » fait évidemment signe en direction de l'école de Francfort, où elle s'articule en particulier au nouvel essor pris par la Théorie critique sous l'impulsion du philosophe Axel Honneth, successeur de jürgen Habermas à la direction de l'Institut de recherches sociales. Chacun sait qu'elle recouvre, dans la définition générale que l'auteur de La Société du mépris en propose, l'ensemble, à vrai dire très 
variable selon les cultures et les états de société, « des relations ou des évolutions sociales qui portent atteinte, pour [...] tous, aux conditions de réalisation de soi » (Honneth, 2006 : 179). Les faits d'inégalité et d'injustice, les misères de condition et de position, les pressions à la performance et à la mobilité, par exemple, ne sont pas seulement, dans cette perspective, des situations pénibles au sein desquelles les sujets sociaux peuvent se trouver plongés et qui resteraient au fond extérieures à leur sphère mentale et corporelle, mais des agents pathogènes susceptibles de faire intrusion dans leur sphère intime et leur for intérieur, d'y provoquer des troubles spécifiques et de placer ceux qui en sont atteints - à différents degrés, nul sans doute n'y échappant - dans un rapport névrotique à eux-mêmes autant qu'à l'ensemble des univers collectifs dont ils relèvent. Parler de pathologies sociales de la communication, au sens où cette dernière serait à la source de pathologies particulières dans ce registre, relève donc bien d'un double paradoxe, dont les auteurs des contributions qui suivent, appartenant à différents secteurs de notre « interdiscipline », ont accepté d'endosser le risque. Paradoxe introduit pour une part au sein de la notion même de « pathologies sociales », en ce que cellesci renvoient à des troubles et à des souffrances au regard de quoi la communication - identifiée sur le modèle de Jürgen Habermas, qui voit en elle le cœur normatif du social, à une active ressource d'entente et de rationalité partagée - est généralement pensée comme de nature à les prévenir ou à les apaiser. Mais paradoxe touchant aussi, pour une autre part, à la communication elle-même ou plus exactement à ce qu'il est socialement convenu de nommer « la communication », appellation mal contrôlée à laquelle il faudrait, dans tout ce qui suit, conserver en pensée les guillemets dont elle est ici entourée.

II est banal aujourd'hui d'observer que sous le vocable de communication se rangent une grande variété de domaines et de niveaux d'analyse, de phénomènes et de points de vue, de terrains d'investigation et de corpus théoriques, de concepts et de méthodes. II est moins banal de remarquer que s'y entremêlent aussi une dimension descriptive/constative - par exemple: « on ne peut pas ne pas communiquer », suivant l'adage de Paul Watzlawick - et une dimension prescriptive : il faut communiquer, tu dois te tenir informé, tu dois être en phase avec ton temps et en interaction harmonieuse avec les autres, etc. Bref, la communication est à la fois un objet (un complexe d'objets très divers) et un discours sur cet objet (sur des objets quelquefois substitués l'un à l'autre pour les besoins de la cause) ; à la fois un monde habité (en tant que matrice du social) et une assignation à résidence dans ce monde ; à la fois une discipline définie au point d'articulation de différents secteurs du champ des sciences humaines comme du champ des sciences appliquées et l'un des secteur de la production marchande (ou un secteur auxiliaire à d'autres champs de cette production); à la fois une valeur doucement 
obligatoire et un travail d'imposition du consentement à cette valeur ; à la fois un ensemble de pratiques offertes à la problématisation scientifique et un faisceau d'enjeux proprement idéologiques. Sous ce dernier rapport, il est frappant de constater que la communication, réduite à un mot fétiche autant qu'à un réflexe d'intoxication, tend à servir à la fois de panacée et d'alibi dans le discours politico-médiatique dominant; ainsi lorsqu'on voit éditorialistes et décideurs portés à interpréter - et à nommer - les conflits sociaux, les résistances au « changement » et aux « réformes », ou encore l'« échec » de tel référendum européen, en termes de « déficit de communication » (ou de « pédagogie ») entre « partenaires » inégalement informés des contraintes et des chances attachées à un « monde de plus en plus complexe » (sur ces différents clichés, voir Durand, 2007). L'auteur d'une anthologie des sciences de l'information et de la communication avait bien raison de mentionner, quoique trop incidemment, que « la phraséologie envahissante [de la communication] constitue l'opium de notre temps » (Bougnoux, 1993 : 10). Phraséologie bien fade et sédative en effet, dans laquelle les vertus psychosociales et politiques de la communication, conçue en termes de « mise en relation », de « connexion », d'《 intercompréhension », de « reliance », etc., n'ont rien à envier, en fait d'explication circulaire, aux bonnes vieilles vertus dormitives prêtées à l'opium. « II y a problème dès que la communication devient la solution à tout », remarquait de son côté Yves Winkin au seuil d'un essai polémique ferraillant à la fois contre des variantes dégradées de modèles théoriques propres aux sciences de la communication - variantes aussi exemplaires que la PNL ou les méthodes de lecture des expressions faciales vulgarisées par un Paul Ekman - et la menace pesant sur ces mêmes sciences, dans un espace universitaire en voie d'hétéronomisation, de se voir enrôlées au service de demandes commerciales, entrepreneuriales ou politiques ; « en revanche, ajoutaitil, on peut se rapprocher de la solution lorsque la communication est appréhendée comme un problème » (Winkin, 2003 : I5).

L'adoption d'un point de vue résolument critique sur la communication dans ses apories théoriques, ses définitions et représentations de sens commun ou bien encore l'eschatologie et la théologie très positive dont elle s'enveloppe à l'heure du recul supposé des « idéologies » et de l'effondrement apparent des grands Tiers symboliques - n'est pas chose neuve bien sûr, ainsi qu'en témoigne l'opération de démontage des constructions savantes et des utopies qui les soutiennent entreprise il y a plus de vingt ans par Lucien Sfez dans son imposante Critique de la communication (1988), puis dans son Dictionnaire critique de la communication (1993), avant d'être prolongée dans Technique et idéologie (2002). Et sans doute faut-il avoir à l'esprit que cette critique ne met pas toujours en aussi bonne compagnie et se rappeler, entre autres choses, que c'est en ayant recours à une métaphorique très lourde, filant sur un 
même thème obsédant les motifs de la « suggestion », de la « contagion » ou de la « contamination », qu'un psychologue doublé d'un idéologue tel que Gustave Le Bon (1895) avait pensé, dans la seconde moitié du $\mathrm{XIX}$ siècle, la propagation des affects et des sentiments constitutive de «l'âme des foules », organismes à part entière dans lesquels la promiscuité des corps conduirait invariablement à l'échauffement des esprits et à l'abandon de tout libre arbitre individuel. Vision réactionnaire d'une communication productrice d'hystérie collective et donc pensée précisément, pour la première fois, semble-t-il, en tant que pathologie sociale à l'œuvre aussi bien dans les mouvements de masse que dans la démocratie du suffrage universel. Et sans doute aussi ne faut-il pas passer sous silence les gratifications symboliques et la prime philosophique dégagées par les postures prenant sur un objet commun un point de vue ostensiblement réfractaire au sens commun. Adorno et Horkheimer, dans La Dialectique de la raison (1944), ou plus récemment Paul Virilio, dans Cybermonde. La Politique du pire (1996), ont ainsi adopté sur l'industrie du divertissement médiatique, pour les premiers, ou sur les changements de régime et les accélérations dans l'espace techno-politique, pour le second, un point de vue aux antipodes des visions lénifiantes ou laudatives, faisant valoir comment la raison émancipatrice en vient, dans une culture technologiquement administrée, à se retourner rationnellement en son contraire-l'aliénation, le conditionnement des consciences, la mystification, la retombée dans la pensée magique et le temps immobile de l'éternel retour du même - ou comment l'interconnexion généralisée des réseaux d'information, grosse d'une catastrophe informationnelle planétaire, sonne virtuellement le glas de la démocratie par excès de contrôle sur les consciences et de vitesse dans la circulation des données. Vision élitiste, en surplomb, donc minoritaire ; foncièrement discutable, assez irritante, sans doute, mais moins exaspérante et en tout cas moins chargée d'effets sur les pratiques sociales ou les choix politiques que les refrains, slogans et formules semi-savantes dont sont aujourd'hui tissés, dans leur redondance majoritaire, les discours sur la « société de l'information », l'« économie immatérielle » ou la « société de la connaissance ».

Au premier tome de ses Mémoires, évoquant le mythe de l'Amérique qui déjà s'est répandu dans les meilleurs esprits, Talleyrand (1967: 7I) soutient que « l'esprit de destruction accourt dans tous les lieux où les communications deviennent plus faciles $\gg$. Et à peu près au même moment, vers 1830, Chateaubriand (1848, t. || :662) - par ailleurs perplexe et prophétique au sujet des « chemins modernes de l'Allemagne », lesquels « serviront seulement à la guerre ; vomitoires à l'usage de nouveaux Barbares qui, sortant du nord avec l'immense train des armes à feu, viendront inonder des régions favorisées de l'intelligence et du soleil »considère avec une féroce ironie ceux de ses contemporains qu'il voit tout affairés à « niais[er] béatement avec des canaux, des chemins de fer, 
des tripotages d'art, des arrangements de lettres ; monde de machines, de bavardage et de suffisance nommée société modèle » (ibid. : 527). « Je ne me laisse pas éblouir, écrit-il encore, par des bateaux à vapeur et des chemins de fer ; par la vente des produits des manufactures et par la fortune de quelques soldats français, anglais, allemands, italiens enrôlés au service d'un pacha : tout cela n'est pas de la civilisation » (I 848, t. I. : 877). Propos d'esprits chagrins, bien dans le ton de ceux qui vieillissent au bord d'un monde qui les abandonne. Expressions évidentes d'une humeur réactionnaire et d'un romantisme réactionnel à mettre évidemment au compte de la commune résistance du prince de Bénévent et de l'auteur des Mémoires d'outre-tombe à une modernité inséparablement technique et politique. Au moins ont-elles pour intérêt, ces prises de position qui nous paraissent d'un autre âge, de remettre à l'esprit, pour peu que l'on y songe, ce que l'idéologie communicationnelle de notre temps conduit à faire oublier ou méconnaître, à savoir que la communication, entendue au pluriel des « communications » ou au singulier qui la dilue en bain anthropologique ou en phénomène social, est - est aussi - une technique de pouvoir et que, dans sa définition la plus matérielle, elle se confond, des réseaux routiers de la Rome antique aux autoroutes de la moderne Allemagne et du télégraphe optique à l'internet, avec des systèmes d'intervention, de surveillance et de contrôle : militaire à l'origine et liée au pouvoir d'État avant d'être mise au service des réseaux économiques et des échanges commerciaux, puis vouée au trafic non moins marchand des immatériaux de l'information et du divertissement. Cette modernité métamorphosée jusqu'à nous en fait de nature ou bien en nouveauté providentielle, il n'est pas inutile de l'historiser de la sorte : comme toute autre, elle se reconnaît à l'amnésie de ses origines autant qu'à l'illusion qu'elle entretient de répondre à une poussée sans autre appui que sa propre force de surgissement et d'évidence. Ainsi, il est peu douteux que Chateaubriand, à travers la « société modèle » qu'il prend pour cible, vise à mots à peine couverts la doctrine « industrialiste » des saint-simoniens, axée sur une théorie des réseaux de communication, telle qu'elle fait tache d'huile entre 1830 et 1840 dans les milieux des progressistes en attendant de se trouver convertie du côté de la technocratie d'État au cours du Second Empire, sous l'impulsion d'un Michel Chevalier, premier titulaire de la chaire d'économie politique du Collège de France et rallié dès 1851 au Prince-Président, dont il se fera le conseiller en la matière. Occasion de rappeler que les développements du système des transports et des technologies de la communication ont été escortés, au fil de ce siècle dont le nôtre reste à tant d'égards l'héritier, par une mystique de l'association et de la paix universelles réalisables au moyen des réseaux (voir Musso, 1997 ; 2003) ; occasion aussi de faire valoir que cette mystique a eu pour contrepartie paradoxale, et comme son répondant en négatif dans les dispositifs politiques autant que dans les structures mentales, la progressive mise en place des conditions de 
possibilité d'une société fragmentée, sérialisée, et articulée - sur fond de dissolution, à tout le moins virtuelle, des groupes et des solidarités de classe - autour de la fiction d'un corps social composé d'un ensemble de sujets abstraits et universels, substituables et additionnés les uns aux autres : fiction dont le principe de l'isoloir au moment des scrutins, à la fois technique de secret et technique d'isolement, apparaîtra à la veille de la Première Guerre mondiale comme la réalisation concrète et symbolique à la fois (voir Garrigou, 1998). L'adoption, entre autres importations, de la pratique américaine des sondages confirmera aux yeux d'un Sartre que la « civilisation technique », si elle est une civilisation « de la généralité », implique par cette généralité même «l'interchangeabilité des individus », « unités juxtaposées » qu'il convient « de lier [...] en une unité organique $\gg$ :

« C'est à quoi tendent, écrira-t-il, les mass media, best seller, book of the month, best record, Gallup, Oscar, etc. Il s'agit de présenter à l'exemplaire isolé l'image de la totalité. Naturellement on n'obtiendra que la majorité. Mais la majorité est donnée comme l'expression de la totalité. [... L L'opinion demeure dehors, elle est le point idéal et transcendant de jonction de toutes les consciences, mais précisément parce qu'elle est un transcendant, elle isole chaque conscience en face d'elle-même, sans lui laisser de mots ni de concepts pour s'exprimer » (Sartre, 1983:93)'.

Les rêveries de l'association universelle, utopie censée se réaliser par la magie politique des grands réseaux et des communications, ont ainsi pour ligne de fuite l'image d'une société penchant en tout ou en partie vers l'anomie - cette anomie dont Jean-Marie Guyau forgera le concept dans son Esquisse d'une morale sans obligation, ni sanction (1885) afin de définir la morale adéquate à un temps caractérisé par l'effondrement des grands principes d'autorité (la religion, l'État, la science), et qu'Émile Durkheim ( 897 : 264-3 I I), dans son étude sur Le suicide, renverra quant à lui aux pathologies suscitées par les situations de crise et, plus généralement, par les états de société condamnant à l'indétermination et à une perpétuelle mobilité ceux qu'une trop grande division du travail isole les uns des autres au prix d'une perte du sentiment de leur interdépendance.

Ce retour trop cursif à l'histoire, invitation à une généalogie des rapports d'implication réciproque existant aussi bien entre technique et mythe technologique qu'entre développement des communications et processus de sérialisation sociale, n'avait pas seulement pour objet général de dissiper l'euphorie des représentations et des notions banalement

\footnotetext{
L'auteur de La Société du spectacle résumera tout cela, à sa manière, en une forte formule : «L'isolement fonde la technique, et le processus technique isole en retour » (Debord, 1967: 21). Entendons, pour notre propos et contre les mythes d'appui des technologies de la communication, qu'il y a boucle récursive entre ces technologies, censées relier, et l'isolement des sujets sociaux.
} 
associées à la communication et, par là, d'aménager la place d'une approche sous plusieurs angles des pathologies susceptibles de lui être liées contrairement aux idées les plus reçues. II s'agissait aussi d'installer I'horizon rétrospectif au regard duquel l'article de David Forest, qu'on lira plus loin, prend une part de son relief. « L'intelligence collective », que son propos passe au scalpel, est en effet l'un des nouveaux poncifs ayant fait intrusion dans l'imaginaire utopique de la communication, exemple supplémentaire de ces prophéties séculières, frottées de ferveur mystique autant que de froid utilitarisme, dont l'escorte discursive faite aux « nouvelles technologies » est coutumière et au sujet desquelles il a déjà fait paraître un solide ouvrage (Le Prophétisme communicationnel, 2004). Cette expression, rapidement intégrée au technolecte des journalistes et dont le lancement a coïncidé en France avec l'entrée de l'internet dans les usages, chacun sait qu'on la doit à Pierre Lévy, promoteur d'une « anthropologie du cyberespace » et d'une vision de l'humanité où, aux monades individuelles propres aux sociétés quadrillées, succéderaient les nomades en libre circulation dans un « cerveau planétaire »: « Les "autoroutes de l'information" et le "multimédia" [...] accroîtrontils le fossé entre les riches et les pauvres, les exclus et les branchés ? C'est effectivement, concédait-il, un des avenirs possibles. Mais si nous mesurons à temps l'importance des enjeux, les nouveaux moyens de communication pourraient aussi renouveler profondément les formes du lien social dans le sens d'une plus grande fraternité, et aider à résoudre les problèmes dans lesquels se débat aujourd'hui l'humanité » (Lévy, 1994: 9). Air connu : le franchissement d'une nouvelle étape dans l'hominisation par l'interconnexion planétaire des réseaux et des ordinateurs recycle un vieux fonds macluhanien, lui-même alimenté par le télescopage du registre scientifique et du registre religieux si caractéristique de la pensée deTeilhard de Chardin, comme aussi, on l'a vu, des prédications saint-simoniennes sur la physiologie commune des réseaux matériels et des réseaux spirituels. Encore le propos de David Forest n'est-il pas tant généalogique que de suivre le cheminement de la notion d'« intelligence collective » à travers un ensemble de discours à teneur politique, économique ou juridique et qui, placés de Michael Hardt et Toni Negri à Yann MoulierBoutang sous le signe d'un « capitalisme cognitif », articulent évocation de « nouvelles réalités anthropologiques », promotion d'une « société contractuelle », apologie de la « déréglementation » et méfiance à l'égard des limitations imposées par la propriété intellectuelle à la circulation des œuvres dans une économie de la mobilité et de l'abondance. Par où l'on voit que la notion d'« intelligence collective » et ses avatars personnages conceptuels dont la plasticité et l'ubiquité réconcilient anarcho-communistes, libéraux-libertaires et partisans du logiciel libre n'apparaissent pas seulement comme propagande publicitaire au profit des industries de l'électronique et des mondes du virtuel, mais s'ajustent assez étroitement à la vulgate néo-libérale telle qu'elle s'est répandue, par 
divers canaux, dans les années 80-90, avec son appareil lexical lourd de conséquences sur les structures politiques et sur la santé psychique des individus : mobilité, évaluation, créativité, innovation, fluidité, responsabilité, flexibilité, etc., modes d'être au social ou formes de représentations de la société dont la communication, sous paradigme électronique, constitue bien évidemment l'un des vecteurs performatifs. Pathologie de la communication ? Oui, selon David Forest, si l'on entend celle-ci « non pas comme l'envers d'une communication "saine", mais plutôt comme [un] jeu permanent entre visibilité et invisibilité [d'un] symptôme affectant un corps social travaillé en profondeur par les idéologies de la communication ». Des idéologies encastrées, comme elles le sont toutes, dans une vision politique et économique du monde, qu'elles dotent d'un puissant pouvoir d'imposition, mais aussi de construction de la réalité sociale - avec les dégâts que l'on sait et qu'un Axel Honneth (2006 : I8I) avait en tête lorsque, dans son entretien de 2001 avec Olivier Voirol, le philosophe préconisait une « analyse de la société orientée vers le diagnostic des pathologies sociales avec l'aide du concept de reconnaissance $[. .$.$] pour être en mesure, précisait-il, de montrer que$ l'évolution du capitalisme néo-libéral actuel va dans une direction où les conditions d'autoréalisation seront, pour nous tous, considérablement meurtries - par exemple à travers les tendances à la marchandisation, les tendances à détruire les relations privées, ou les exigences accrues de gestion de l'identité »).

Dans le cas de «l'intelligence collective » et de ses ramifications, la pathologie en jeu, toute puissante qu'elle soit, demeure en quelque sorte extérieure à l'acte de communication : la genèse d'un tel concept, son frayage politique, son adéquation à un contexte technique et économique, sa rentabilité idéologique tiennent d'une charge sémantique, certes opportuniste et très ciblée, plus que d'une pragmatique fondamentale. C'est sur ce dernier plan que Christine Servais et Véronique Servais, respectivement spécialistes de l'esthétique des médias et de l'anthropologie de la communication, ont fait en revanche porter leur réflexion, raison pour laquelle il a paru significatif d'installer celle-ci au seuil du dossier. Les représentations communes de la communication associent sa « réussite » à la transmission efficace d'un message, donc débouchant sur des effets conformes à l'intention de l'émetteur, et à l'intercompréhension de l'émetteur et du récepteur, supposés non seulement maîtriser un même code, mais appartenir à un même univers de représentation. C'est cette idée reçue, dont dérivent tant de modèles usuels de la communication et dont procède le privilège excessif accordé à la notion de « consensus », que Christine et Véronique Servais soumettent à interrogation, à la lumière des systèmes de communication homme/animal (en l'occurrence tels qu'ils se donnent à observer dans les situations de « rencontres enchantées » entre hommes et dauphins 
sauvages) et à l'aide des concepts de « différend », de « mésentente » et dans une moindre mesure de « différance », empruntés tour à tour à Jean-François Lyotard, Jacques Rancière et Jacques Derrida, concepts qui tous prennent en charge, à quelque niveau, la problématique de l'altérité et de reconnaissance de cette altérité dans l'acte et la situation de communication. La thèse ici défendue, au prix d'un examen de témoignages rapportant de telles rencontres et d'un entrecroisement très serré de ces concepts philosophiques avec les travaux du linguiste Antoine Culioli et de l'anthropologue Franco La Cecla, ou encore avec les apports de Gregory Bateson et de l'école de Palo Alto, est que penser la communication en termes de transmission efficace et de monde partagé revient en quelque sorte à lui attribuer la manipulation intentionnelle pour idéal légitime et à oblitérer le fait que le malentendu pourrait bien en constituer, paradoxalement, la structure fondamentale en tant qu'il représente la condition d'émergence et de maintien de l'altérité au sein de l'interaction. Compter sans cette structure expose, selon Christine et Véronique Servais, à des conséquences psychopathologiques - à travers le contrôle du partenaire, la négation de son altérité - ou politiques par imposition de l'idiome du dominant : « La volonté de supprimer le malentendu, de le résoudre en s'en rapportant à une règle, est toujours le moment où émerge la domination ; celle-ci se poursuivra tant que le dominé ne pourra se faire entendre dans l'idiome du dominant, prendre la parole depuis une place qui ne lui est pas reconnue, auquel cas l'interaction se transformera alors en conflit proprement politique ». Et d'argumenter en faveur d'une conception de la communication fondée sur une pluralité effective ; non tant sur un monde partagé, renvoyant à une hégémonie de représentations et de langage, que sur une ligne de partage passant aussi bien entre les partenaires de l'interaction qu'en chacun d'eux, comme forme de distance et de différence de soi à soi, « indispensable pour qu'un rapport à l'autre soit seulement possible ».

La censure relève-t-elle d'une distorsion pathologique du processus de communication sous contrainte politique, morale, économique ou religieuse, ou bien en constitue-t-elle une composante essentielle ? La question a de quoi étonner à deux titres, dès lors que la censure, du moins sous sa forme préventive, semble avoir disparu de nos régimes démocratiques, voués à la transparence et à la libre communication des idées et des œuvres, et dès lors qu'il semble tomber d'autre part sous le sens qu'entre censure et communication les rapports ne sauraient être que de pure extériorité, pouvoir autoritaire de répression ou du moins de contrôle pour l'une, effort constant en vue d'une expression sans entraves pour l'autre. Historien de la presse, Laurent Martin révoque pour commencer l'idée selon laquelle, si l'on en croit le grand récit émancipateur avec lequel se confond l'histoire des sociétés libérales, la censure se serait effacée de notre espace public ; elle s'est bien plutôt 
déplacée, comme par paliers, en suivant les transformations du système médiatique en regard du public élargi susceptible d'être atteint, pour passer graduellement du livre imprimé ( $\left.X V\right|^{e}-X V I I l e$ siècles), au théâtre et à la presse périodique ( $X V\left|\|^{e}-X\right| X^{e}$ siècles), puis auX médias audiovisuels ( $X X^{e}$ siècle) et enfin, de nos jours, au réseau internet; d'autre part, si la censure s'est largement déplacée aussi de l'amont vers l'aval pour sanctionner les abus de la liberté d'expression, sa forme préalable reste en activité au sein de la commission de contrôle des films cinématographiques instituée dès 1919 dans une France « dont la législation reste si contraignante qu'il est "de coutume de dire, chez les juristes spécialisés, que si un "message" est diffusable en France, il l'est impunément partout dans le monde ou presque"» (Pierrat, 2008 : 19); enfin, estompée, adoucie, toujours possible, la censure n'en reste pas moins efficace sous les espèces qu'elle stimule de l'autocensure et de l'autorégulation. Parler, d'autre part, d'une censure intégrée au processus de communication lui-même, c'est faire place à l'ensemble des travaux qui, inspirés par Roland Barthes, Michel Foucault ou encore Pierre Bourdieu, mettent en lumière les processus de filtrage de la pensée imposés tant par l'ordre de la langue et des discours que par les « formations de compromis », le travail d'euphémisation verbosociale, les mises en forme et aux normes auxquelles l'expression se voit tenue par les structures et attentes propres au champ dans lequel et pour lequel elle se formule : « censure structurale » ou « censure invisible », propice à l'orthodoxie des opinions et dont la conséquence paradoxale serait, au fond, de faire passer la censure du statut de pathologie à celui de forme normale d'une communication vouée à intérioriser les contraintes auxquelles elle se doit de répondre pour être acceptée, reçue, comprise, sinon pensée en amont. Le risque est grand cependant d'instituer la censure, si elle est partout et toujours déjà à l'œuvre, en force si diffuse et permanente qu'elle en devient inidentifiable, d'écraser les régimes divers qu'elle peut adopter et de désarmer la critique en la rendant par avance inopérante. « II peut être préférable, pour l'analyse scientifique et politique, argumente Laurent Martin, de réserver le terme de censure à des phénomènes dûment certifiés d'intervention autoritaire d'un tiers dans la communication entre un émetteur [...] et un récepteur » et d'encourager la mise en œuvre, contre cette pathologie effective, d'une thérapeutique vigilante allant de la mobilisation des acteurs concernés à l'édiction de règles prévenant les abus du censeur.

Ce sont d'autres pathologies encore qu'Élise Vandeninden, chercheuse dans le domaine de la médiation culturelle, porte au jour à partir d'une enquête sur les pratiques d'art-thérapie. Elle y débusque les tensions entre les différentes conceptions dont celles-ci font l'objet, non sans mettre en évidence un investissement commun aux divers praticiens : la création est un « outil » de médiation, soit à l'intérieur d'une institution de soin, soit avec l'extérieur. Aussi est-ce une approche « grammaticale », 
à distance de toute velléité normative, qu'elle propose dans le sillage de Luc Boltanski (2004) et sur la base d'un corpus d'ouvrages sur l'artthérapie - approche qui lui permet de tracer les contours de grands modèles d'activité articulant la relation entre le destinateur, le « tiers médiateur » et les divers destinataires (individuels ou collectifs). Elle montre également qu'à ces types de processus correspondent autant de « vertus thérapeutiques » supposées. C'est bien sur ces modélisations en effet qu'ÉliseVandeninden fait porter son enquête auprès d'un échantillon d'artthérapeutes, au moyen d'une sociologie toute pragmatique, s'attachant à rendre compte d'une véritable « mise à l'épreuve » par l'expérience en contexte. L'intérêt de ses résultats réside pour l'essentiel par ce qu'ils font émerger en fait de décalages entre les grands modèles inspirateurs et leur mise en œuvre, mettant en lumière quelquefois, chez tels praticiens, un doute touchant à la réalité de la vertu thérapeutique. Certains d'entre eux revendiquent même des « objectifs purement esthétiques ». Ces hiatus sont interprétés à la lumière de la trajectoire et de la position des agents dans le champ artistique et médical, ce dernier étant dominant. De ces analyses surgissent des hypothèses fortes voulant que le patient soit placé par l'art-thérapeute dans une situation paradoxale : « l'art-thérapie "médicale", qui se veut médiation, risque d'aboutir en définitive à la création d'une véritable pathologie de la communication : celle qui place le patient dans ce que, à la suite de Gregory Bateson, Paul Watzlawick, Janet Helmick Beavin et Don D. Jackson (1967 : 195-196) ont appelé une "double contrainte" ». Quant à la médiation fondée sur un pôle davantage artistique - mais marginal -, elle conduit à introduire un autre langage dans l'institution et à modifier le rôle du patient : «Utiliser l'art "pour l'art" en psychiatrie revient-il à se situer dans la critique "interactionniste" de la maladie mentale; perspective selon laquelle "l'attaque contre une hiérarchie commence par une attaque portant sur les définitions, les étiquettes et les représentations conventionnelles de l'identité des personnes et des choses" » (Becker, 1963 : 229). On comprend par là que réserver un regard communicationnel à l'art-thérapie débouche sur une remise en cause de ses fondements.

Si notre dossier se boucle - en fin nécessairement ouverte - avec l'article de Thomas Heller sur « La reconnaissance et le gouvernement des salariés », c'est que celui-ci rapatrie en quelque sorte les pathologies du social dans un contexte et sous un angle de vue qui leur sont naturellement associés, à savoir les souffrances psychosociales endurées dans ce haut lieu de contrôle et de dressage anthropologique que l'entreprise représente aujourd'hui. En prenant appui sur les travaux fondateurs d'Axel Honneth, l'auteur s'y emploie à mettre en évidence certains des processus par lesquels s'opèrent, dans ce contexte, « des formes de domination articulées à la quête identitaire et au désir de réalisation de soi ». Partant du postulat que, dans le monde salarial, la 
reconnaissance - ou affirmation des qualités d'un individu ou d'un groupe - est revendiquée dès lors qu'elle vient à manquer, il montre que celle-ci peut aussi être un alibi au service du management et des ressources humaines, et cela afin de favoriser la motivation au travail. Fondant sa démonstration sur des exemples empruntés à l'ouvrage de Christophe Dejours, Souffrance en France. La banalisation de l'injustice sociale (1998), Thomas Heller aborde « le rapport de la communication au mépris ou au déni de reconnaissance ». Mais pour conduire à bien son analyse, il distingue le mépris du déni de reconnaissance, en ce que le premier « ne relève pas de pratiques isolées mais qu'il est érigé en système $[\ldots]$, en stratégie managériale » et que le second « se manifeste notamment par des obstacles aux pratiques de reconnaissance ». Quoi qu'il en soit et à l'instar de Christophe Dejours, l'auteur montre combien la communication qui engage les salariés à se montrer sous leur meilleur jour - ou leur service - participe, au nom de la raison économique, du déni de la réalité du travail. Distorsion qui, à ses yeux, touche de près au mépris. D'où l'hypothèse qu'il formule voulant que la communication, « en tant que pratique professionnelle d'élaboration et de diffusion, a pour conséquence de faire obstacle à la reconnaissance et qu'elle peut être considérée comme une pratique productrice de pathologies sociales ». Et d'en tirer l'idée selon laquelle la reconnaissance s'associe à un procédé managérial consistant à valoriser le salarié en flattant chez lui un rapport satisfaisant à lui-même pour obtenir, « ponctuellement ou durablement, un effet de soumission et/ou de performance ».

Theodor W. Adorno et Max Horkheimer (1944:22) tenaient que « seule une pensée qui se fait violence à elle-même a la dureté nécessaire à la destruction des mythes ». Le dossier que voici n'a pas la prétention d'y atteindre, ni l'ambition, on l'a dit en commençant, de faire le compte rendu exhaustif, au creux du doux consensus qui l'entoure d'ordinaire, des violences plus ou moins symboliques dont la communication peut s'avérer porteuse - moins par ses contenus, ici, que par la structure qui est la sienne et l'efficace qu'elle détient en tant que processus social ou ressource enrobée de mythologie. La variété des objets abordés dans les pages qui suivent, le rythme discontinu du propos, tantôt elliptique, tantôt insistant, la diversité de disciplines convoquant tour à tour le point de vue de l'historien, du sociologue, de l'anthropologue, du juriste, du philosophe ne s'y conjuguent pas moins, il nous semble, pour mettre en question féconde, à l'enseigne de Questions de communication, l'objet cardinal de la revue.

\section{Références}

Becker H., 1963, Outsiders. Études de sociologie de la déviance, trad. de l'américain par J.-P. Briand, J.-M. Chapoulie, Paris, Métaillé, 1985. 
Boltanski L., 2004, La condition fœtale. Une sociologie de l'engendrement et de l'avortement, Paris, Gallimard.

Bougnoux D., 1993, Sciences de l'information et de la communication, Paris, Larousse.

Chateaubriand Fr. R. de, I848, Mémoires d'outre-tombe, tomes I et II, Éd. M. Levaillant et G. Moulinier, Paris, Gallimard, 1951.

Debord G., 1967, La société du spectacle, Paris, Buchet-Chastel.

Dejours Chr., 1998, Souffrance en France. La banalisation de l'injustice sociale, Paris, Éd. Le Seuil.

Durkheim É., 1897, Le suicide, Paris, Presses universitaires de France, 1999.

Durand P., dir., 2007, Les nouveaux mots du pouvoir. Abécédaire critique, Bruxelles, Éd. Aden.

Forest D., 2004, Le prophétisme communicationnel, Paris, Éd. Syllepse.

Garrigou A., 1998, « Le secret de l'isoloir », Actes de la recherche en sciences sociales, 7I-72, pp. 22-45.

Guyau J.-M., 1885, Esquisse d'une morale sans obligation, ni sanction, Paris, Éd. Fayard, 1985.

Honneth A., 1992, La lutte pour la reconnaissance, trad. de l'allemand par P. Rusch, Paris, Éd. du Cerf, 2007.

— 2006, La société du mépris. Vers une nouvelle théorie critique, trad. de l'allemand par O.Voirol, P. Rusch, A. Dupeyrix, Paris, Éd. La Découverte, 2008.

Horkheimer M.,Adorno Th.W., 1944, La dialectique de la raison, trad. de l'américain par E. Kaufholz, Paris, Gallimard, 1974.

Le Bon G., 1895, Psychologie des foules, Paris, Presses universitaires de France, 1995.

Lévy P., 1994, L'intelligence collective. Pour une anthropologie du cyberespace, Paris, Éd. La Découverte.

Musso P., 1997, Télécommunications et philosophie des réseaux. La postérité paradoxale de Saint-Simon, Paris, Presses universitaires de France.

- 2003, Critique des réseaux, Paris, Presses universitaires de France.

Pierrat E., dir, 2008, Le livre noir de la censure, Paris, Éd. Le Seuil.

Sartre J.-P., 1983, Cahiers pour une morale, Paris, Gallimard.

Sfez L., 1988, Critique de la communication, Paris, Éd. Le Seuil, 1992.

- dir., 1993, Dictionnaire critique de la communication, 2 vol., Paris, Presses universitaires de France.

— 2002, Technique et idéologie, Paris, Éd. Le Seuil.

Talleyrand Ch. M. de, 1967, Mémoires ou opinion sur les affaires de mon temps. Tome I : La Révolution, Paris, J. de Bonnot.

Virilio P., 1996, Cybermonde. La politique du pire, entretien avec Ph. Petit, Paris, Éd. Textuel.

Watzlawick P., Helmick Beavin J., Jackson Don D., 1967, Une logique de la communication, trad. de l'américain par J. Morche, Paris, Éd. Le Seuil, 1972.

Winkin Y., 2003, La Communication n'est pas une marchandise, Bruxelles, Éd. Laborl Espace de libertés. 